\title{
$17 \beta$-Estradiol Reduces Nitric Oxide Production in the Guinea Pig Cochlea
}

Authors

Affiliations

\section{U.-R. Heinrich ${ }^{1}$, J. Brieger ${ }^{1}$, C. Striedter ${ }^{1}$, I. Fischer ${ }^{1}$, I. Schmidtmann², H. Li³ , W. J. Mann ${ }^{1}$, K. Helling}

${ }^{1}$ Department of Otorhinolaryngology, Head and Neck Surgery, University Medical Center of the Johannes Gutenberg University Mainz, Mainz, Germany

${ }^{2}$ Institute for Medical Statistics, Epidemiology and Informatics (IMBEI), University Medical Center of the Johannes Gutenberg University Mainz, Mainz, Germany

${ }^{3}$ Department of Pharmacology, University Medical Center of the Johannes Gutenberg University Mainz, Mainz, Germany

\section{Key words \\ - inner ear \\ - organ culture \\ - cytoprotection \\ - lateral wall \\ Q organ of Corti}

received 25.04.2013

accepted 06.05 .2013

\author{
Bibliography \\ DOI http://dx.doi.org/ \\ 10.1055/s-0033-1347200 \\ Published online: \\ June 21, 2013 \\ Horm Metab Res 2013; \\ 45: 887-892 \\ (c) Georg Thieme Verlag KG \\ Stuttgart · New York \\ ISSN 0018-5043
}

\section{Correspondence}

\section{K. Helling, MD}

Department of Otorhinolaryngology, Head and Neck Surgery University Medical Center of the Johannes Gutenberg University Mainz

Langenbeckstrasse 1

55131 Mainz

Germany

Tel.: + 49/6131/17 7361

Fax: +49/6131/17 6637

kai.helling@unimedizin-mainz.de

\section{Abstract \\ $\nabla$}

Intense noise exposure and the application of ototoxic substances result in increased levels of reactive oxygen species (ROS) and reactive nitrogen species (RNS), such as nitric oxide (NO). In order to reduce the free NO concentration in the inner ear under pathological conditions, the use of natural cytoprotective substances such as $17 \beta$-estradiol is a promising therapeutic concept. In male guinea pigs the organ of Corti and the lateral wall were isolated from the cochlea and afterwards incubated for $6 \mathrm{~h}$ in cell-culture medium. $17 \beta$-Estradiol was adjusted in 2 concentrations to organ cultures of the right ears (12 animals per concentration). The left ears were used as controls. The NO production was quantified in the supernatant by chemiluminescence after incubation. Depending on the concentration,

\section{Introduction}

$\nabla$

It has been known for some years that nitric oxide (NO) is not only a versatile key player in cochlear function, but also responsible for inner ear hearing disorders [1-3]. Based on different animal models, it is currently assumed that an increased NO production triggers various inner ear diseases such as acute noise trauma, sudden idiopathic hearing loss, acute tinnitus, and presbycusis [4-8]. Only glucocorticoids which include synthetic dexamethasone have been clinically used in inner ear therapy so far [9-11]. Based on this restricted repertoire of drugs and the limited knowledge about the underlying NO-dependent mechanisms in inner ear pathophysiology, it is of great medical and economic interest to develop new therapeutic strategies against inner ear distress. One strategy might be based on the utilization of the body's own natural substances, such as the sex gonadal steroid hormone $17 \beta$-estradiol.
$17 \beta$-estradiol reduced NO in the organ of Corti by $43 \%(\mathrm{p}=0.015)$ and $46 \%(\mathrm{p}=0.026)$, respectively. In the lateral wall, the NO concentration was reduced by $24 \%$, but without statistical significance $(p=0.86)$. However, when analyzing the association between the 2 cochlear regions for each animal separately, the NO concentrations were lower in nearly all $17 \beta$-estradiol-treated ears compared to controls. In order to demonstrate the flexibility of the organ culture system, the NO donor DETA NONOate and the nitric oxide synthase inhibitors L-NAME and L-NMMA were applied. The electron microscopic analysis revealed a well-preserved cochlear cell morphology after incubation. The ability of $17 \beta$-estradiol to influence the NO production preferentially in the organ of Corti might offer new therapeutic perspectives for inner ear protection.

In different approaches, it was shown that $17 \beta$-estradiol can influence NO production differently depending on the tissue. In the endothelium of blood vessels, a $17 \beta$-estradiol-dependent increase in the endothelial nitric oxide synthase (eNOS) expression or an upregulation of its activity was detected, both resulting in an increased NO concentration [12]. On the other hand, a $17 \beta$-estradiol-dependent downregulation of nitric oxide synthase activity was identified in the uterus and vagina of rabbits [13].

At the cellular level, an estrogen-mediated inhibition of $\mathrm{Ca}^{2+}$ influx via L-type $\mathrm{Ca}^{2+}$ channels was described for murine cardiomyoctes [14] and this attenuated glutamate-induced cell death in a ventral spinal motoneuron and neuroblastoma hybrid cell line (VSC 4.1) [15]. Thus, 17 $\beta$-estradiol was found to fulfill multiple tasks in NO-regulated vertebrate physiology including neuroprotection [16-19]. In addition, clinical and experimental studies have shown that estradiol 
plays an important role in inner ear adaptation in respect to sound perception, especially in females [20].

Despite its known gender-dependent alterations in auditory physiology [20], estradiol has more common, gender-independent functions in the inner ear. The 2 known estrogen receptors (ER $\alpha$ and $E R \beta$ ) were found to be co-expressed in cell nuclei of different cell types in the inner ear in both male and female vertebrates. In the mouse model, both receptors were localized in the cochlea within the nuclei of stria vascularis, in the outer and inner hair cells and in the spiral ganglion cells [21]. Analyzing quantitative differences in immunohistochemical staining intensities, ER $\alpha$ was more dominant in young females than in young males. The expression of both receptors was clearly reduced in old animals of both sexes compared with young ones [21].

Besides receptor-dependent genomic effects, numerous nongenomic effects were identified in different systems summarized by Chow et al. [22]. These effects are faster than the genomic regulated processes and occur within minutes rather than hours [22]. The aim of this animal study was to clarify the still unknown question of whether the application of the neuroprotective agent estradiol can influence NO production within the vertebrate cochlea. Therefore, organ cultures of the organ of Corti and the lateral wall were incubated in culture medium and the NO production was determined in the supernatant by chemiluminescence. Any alterations in NO production and the presumed estrogen-dependent mechanisms are discussed below.

\section{Materials and Methods}

$\nabla$

\section{Animals}

Twenty-four healthy pigmented male guinea pigs (tricolor, Charles River, Sulzfeld, Germany) weighing 200-250 g with good Preyer's reflexes and no evidence of middle ear disease were used in the present study. Up to 6 animals were housed in 1 cage and maintained at a 12:12-h light:dark cycle in the university animal facility. All experiments were conducted in accordance with the German Prevention of Cruelty to Animals Act and were approved by the supervising authorities.

\section{Cochlea preparation and tissue incubation}

Animals were killed by sodium pentobarbital (Narcoren ${ }^{\circledR}$; Hallbergmoos, Germany; $448 \mathrm{mg} / \mathrm{kg}$ body weight) and the bullae were rapidly removed. Thereafter, the organ of Corti and lateral wall were completely removed from the cochlea and incubated separately in culture medium for $6 \mathrm{~h}$ at $37^{\circ} \mathrm{C}$ in a humidified atmosphere of $95 \%$ air and $5 \% \mathrm{CO}_{2}$, which are the widely used conditions for cell cultures and organ cultures [23,24]. The culture medium was a Dulbecco's Modified Eagle's Medium (DMEM, Invitrogen; Karlsruhe, Germany), which was modified by adding $1 \%$ Glutamax (Invitrogen), $1 \%$ penicillin/streptomycin (PAA Laboratories, Pasching, Austria), 1\% ml sodium pyruvate solution (PAA Laboratories) and $10 \%$ inactivated fetal calf serum (PAA Laboratories). Prior to incubation, a volumn of $150 \mu \mathrm{l}$ $17 \beta$-estradiol (Sigma, St Louis, MI, USA) was admitted in 2 final concentrations to cultures of the organ of Corti or the lateral wall of the right ears. The cultures of the left ears were used as controls (group A). Animals of experimental group B $(n=12)$ received $17 \beta$-estradiol in a concentration of $2.5 \mathrm{ng} / \mathrm{kg}$ body weight (bw) corresponding to $10^{-8} \mathrm{M}$ and animals of group $\mathrm{C}$ $(n=12)$ received $17 \beta$-estradiol in a concentration of $2.5 \mu \mathrm{g} / \mathrm{kg}$ bw corresponding to $10^{-5} \mathrm{M}$ after recommendation by others [16].
After incubation, the supernatants were shock-frozen in liquid nitrogen and stored at $-80^{\circ} \mathrm{C}$ until analysis.

\section{NO determination}

The formation of $\mathrm{NO}_{2}^{-}$, the oxidation product of $\mathrm{NO}$, was determined in the supernatants using an NO analyzer (NOA 280i, Sievers Instruments Inc.; Boulder, CO, USA). The oxidation products of NO were measured using a reaction tank containing $2 \mathrm{ml}$ of $0.1 \mathrm{M}$ potassium iodide (Merck; Darmstadt, Germany) and $16 \mathrm{ml}$ of glacial acetic acid (Roth; Karlsruhe, Germany). The reaction of NO with ozone led to the emission of red light and was in direct proportion to the $\mathrm{NO}_{2}{ }^{-}$concentration in the samples analyzed. The NO concentration was determined for the organ of Corti and the lateral wall of untreated controls (group A) after adding a $17 \beta$-estradiol concentration of $10^{-8} \mathrm{M}$ (group $\mathrm{B}$ ) or a concentration of $10^{-5} \mathrm{M}$ (group C).

The influence of NO donors and NOS inhibitors on the NO production in the system used was tested by incubating organ cultures with 3 different substances: the NO donor (Z)-1-2[2aminoethyl)- $N$-(2-ammonioethyl1)amino]diazen-1-ium-1,2diolate] (DETA NONOate, Alexis Biochemicals; Grünberg, Germany) given in 2 concentrations, the NOS-inhibitor $\mathrm{N}^{\mathrm{G}}$ nitro-L-arginine methyl ester (L-NAME; Alexis Biochemicals), and the NOS-inhibitor $\mathrm{N}^{\mathrm{G}}$-monomethyl-L-arginine monoacetate (L-NMMA; Calbiochem Merck Biosciences; Schwalbach, Germany). In detail, the organ of Corti and the lateral wall of 4 ears in each case were incubated with $50 \mu \mathrm{M}$ DETA and $500 \mu \mathrm{M}$ DETA solutions demonstrating NO upregulation (positive controls) and with $500 \mu \mathrm{M}$ L-NAME and $200 \mu \mathrm{M}$ L-NMMA demonstrating NO downregulation (negative controls).

\section{Electron microscopy}

Six hours after incubation in cell culture medium, the cochlear tissues were taken out and fixed for at least $12 \mathrm{~h}$ in a mixture of $4 \%$ freshly made paraformaldehyde, $0.1 \%$ glutaraldehyde, and $0.2 \%$ picric acid dissolved in $0.1 \mathrm{M}$ sodium phosphate buffer (PBS, pH 7.4). Subsequently, the tissues were dehydrated and embedded in the hydrophilic methacrylate resin, London Resin (LR) White. After hardening the resin for 3 days at $60^{\circ} \mathrm{C}$, the samples were cut with a microtome (Ultracut, Reichert-Jung/Leica) and placed on nickel grids. Staining was performed with uranyl acetate and the cell morphology was analyzed by a ZEISS 906 electron microscope (Oberkochen, Germany).

\section{Statistical approaches}

We used a mixed linear model to analyze the association of estradiol treatment and $\mathrm{NO}_{2}^{-}$production in the organ of Corti and the lateral wall. Animal effect was considered as a random effect, thereby taking possible dependencies due to repeated use of animals into account. The mean values and the standard deviations (SD) are presented in the corresponding graphs and in the text; the p-values are included in the text. All tests were used in an explorative sense and therefore $\mathrm{p}$-values of these tests must be considered descriptive. Statistical analyses were performed using SAS 9.2 (SAS Institute, Inc., Cary, NC, USA) and the mixed linear model was fitted using PROC MIXED.

We investigated the association of $\mathrm{NO}_{2}{ }^{-}$in the organ of Corti and the lateral wall for each of the treatment groups. $\mathrm{NO}_{2}{ }^{-}$values were in the "low" group if their values were closer to the minimum than to the maximum; otherwise they were in the "high" group. The measurements are displayed in a scatter plot below and the lines separating "low" and "high" are shown. 


\section{Results \\ $\nabla$}

\section{Cell morphology}

The electron microscopic analysis revealed a well-preserved cell morphology after the $6 \mathrm{~h}$ incubation period ( $\boldsymbol{0}$ Fig. 1 ). In the extracted tissue of the organ of Corti, the Hensen cells could be clearly identified because of their marginal position and their specific cellular morphology. The microscopic fine structure revealed an undamaged cell membrane and there was no evidence for apoptotic or necrotic processes or vacuolization ( $\odot$ Fig. 1). The partly-cut microvilli that protrude into the endolymph of the Scala media could be seen as well as the homologous tight junctions at the basolateral cell-side ( $\bullet$ Fig. 1).

\section{Nitric oxide content in the cochlea}

The $\mathrm{NO}_{2}{ }^{-}$production was lowered by $17 \beta$-estradiol in cultures of both the organ of Corti and the lateral wall ( $\bullet$ Fig. 2 ). In the organ of Corti, $17 \beta$-estradiol reduced the mean $\mathrm{NO}_{2}{ }^{-}$production in a dose-dependent manner by $43 \%$ or $46 \%$, respectively, compared with untreated controls. In the lateral wall, 17ß-estradiol led to an $\mathrm{NO}_{2}{ }^{-}$reduction of about $24 \%$.

In detail: In the organ of Corti, a mean $\mathrm{NO}_{2}{ }^{-}$content of $293 \mathrm{nM} \pm 172 \mathrm{nM}$ SD was measured in untreated animals (group A). After $17 \beta$-estradiol application, the average $\mathrm{NO}_{2}{ }^{-}$content was reduced to $168 \mathrm{nM} \pm 147 \mathrm{nM} \mathrm{SD}$ (group B, estradiol $10^{-8} \mathrm{M}$ ) or to $158 \mathrm{nM} \pm 107 \mathrm{nM} \mathrm{SD}$ (group C, estradiol $10^{-5} \mathrm{M}$ ), respectively. This reduction was evident when comparing group $\mathrm{A}$ and group $B(p=0.015)$ and when comparing groups $A$ and $C$ $(p=0.026)$. The difference between groups $B$ and $C$ was within random variation $(\mathrm{p}=0.857)$.

In the lateral wall, a mean $\mathrm{NO}_{2}{ }^{-}$content of $489 \mathrm{nM} \pm 224 \mathrm{nM} \mathrm{SD}$ was determined for the untreated control group (group A). $17 \beta$-Estradiol reduced the $\mathrm{NO}_{2}{ }^{-}$content to $373 \mathrm{nM} \pm 160 \mathrm{nM} \mathrm{SD}$ (group B, estradiol $10^{-8} \mathrm{M}$ ) or to $375 \mathrm{nM} \pm 226 \mathrm{nM} \mathrm{SD}$ (group C, estradiol $\left.10^{-5} \mathrm{M}\right)$. However, all differences were within random variaton ( $p=0.131$ comparing groups $A$ and $B, p=0.125$ comparing groups $A$ and $C$ ).

The up- and downregulation of NO production within the cochlear tissues was demonstrated using the NO donor DETA and the 2 NOS inhibitors L-NAME and L-NMMA. When DETA was given at a low concentration ( $\Theta$ Fig. 3a), a significant increase in NO production was detected in the organ of Corti $(p=0.039)$ compared with controls. No effect was seen in the lateral wall $(p=0.526)$. After application of the high DETA concentration, the NO production was clearly increased in the organ of Corti and lateral wall $(\mathrm{p}<0.001)$. Comparing the low and high concentrations, significant differences were found for both the organ of Corti and the lateral wall $(\mathrm{p}<0.001)$.

A downregulation of NO production was observed after application of L-NAME or L-NMMA ( $\bullet$ Fig. $\mathbf{3 b}$ ). In the organ of Corti, the NO production was statistically significantly reduced by L-NAME $(p=0.028)$ and by L-NMMA $(p=0.020)$. Also in cultures of the lateral wall, the NO production was reduced by L-NAME $(p=0.032)$ and by L-NMMA $(p=0.036)$. There was no statistically significant difference in the effect on NO production by L-NAME and L-NMMA in either the organ of Corti $(p=0.598)$ or the lateral wall $(\mathrm{p}=0.809)$.

\section{Inter-animal variation of NO production}

When comparing the individual $\mathrm{NO}_{2}{ }^{-}$production of the organ of Corti and the lateral wall of all experimental groups, differences between $17 \beta$-estradiol-treated ears and controls became evident

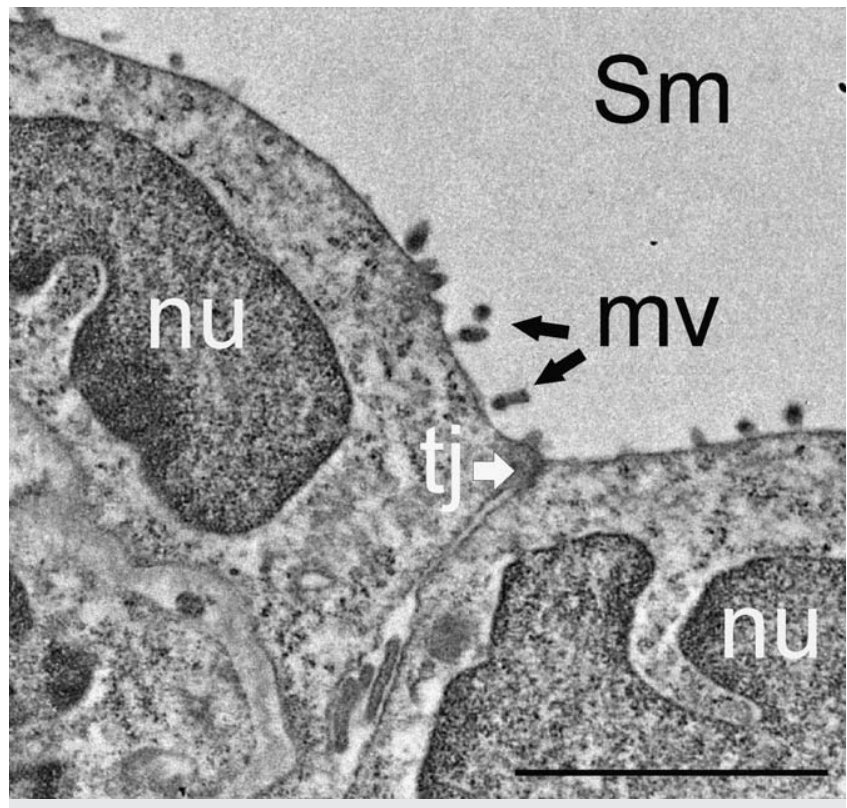

Fig. 1 Electron microscopic image of the Hensen cells. $6 \mathrm{~h}$ after incubation in culture medium, the cellular morphology is well-preserved without any evidence of necrotic processes. nu: nucleus; mv: microvilli; Sm: Scala media; tj: tight junction. Scale bar $=2 \mu \mathrm{m}$.

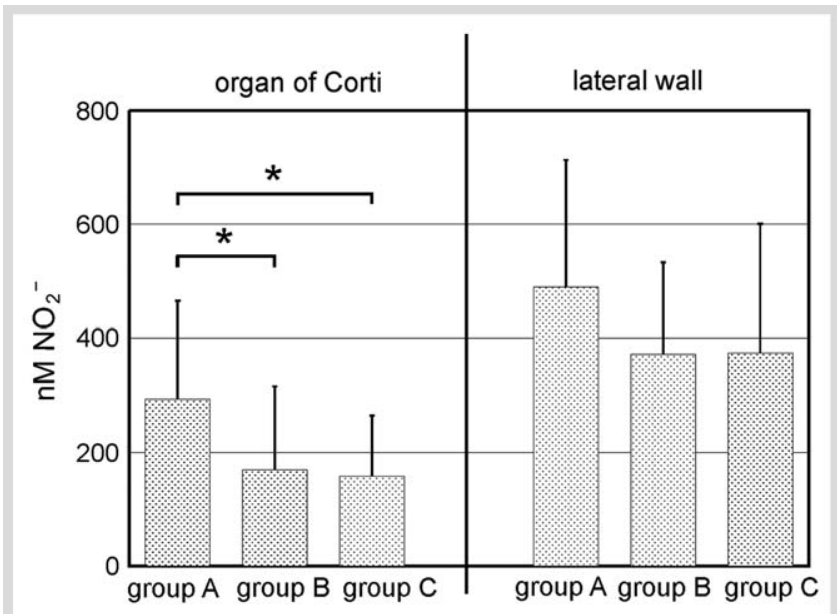

Fig. 2 Concentration of $\mathrm{NO}_{2}{ }^{-}$in $\mathrm{nM}$ (Columns: mean $\pm \mathrm{SD}$ ) measured in the supernatant of cultures of the organ of Corti and the lateral wall. $A$ statistically significant reduction of $\mathrm{NO}_{2}{ }^{-}$production by $17 \beta$-estradiol was identified in the organ of Corti when comparing the control ears (group A) with group B and group C. Statistically significant changes are marked by the asterisks $\left({ }^{*} \mathrm{p}<0.05\right)$.

by dividing the amount of $\mathrm{NO}_{2}{ }^{-}$production into ranges of low and high values ( $\odot$ Fig. 4). After application of $17 \beta$-estradiol, a reduced $\mathrm{NO}_{2}{ }^{-}$production was identified in 21 out of 24 ears (87.5\%). In these ears, the $\mathrm{NO}_{2}{ }^{-}$production was below $468 \mathrm{nM}$ in the lateral wall and below $328 \mathrm{nM}$ in the organ of Corti. A higher $\mathrm{NO}_{2}{ }^{-}$production was only found in 3 ears (12.5\%). In controls, a reduced $\mathrm{NO}_{2}{ }^{-}$production below these specified values was only found in 9 ears (39\%), whereas in 14 ears the $\mathrm{NO}_{2}{ }^{-}$production was above these values (61\%). 


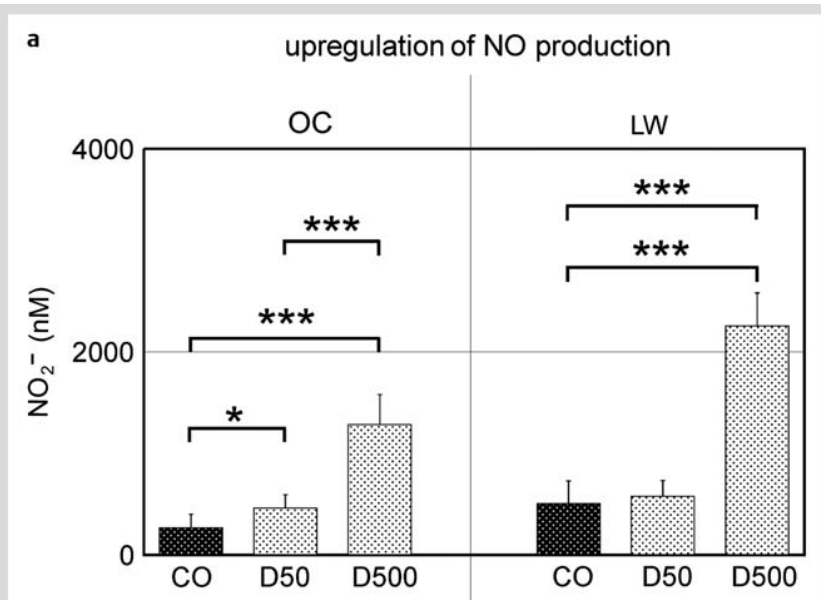

b

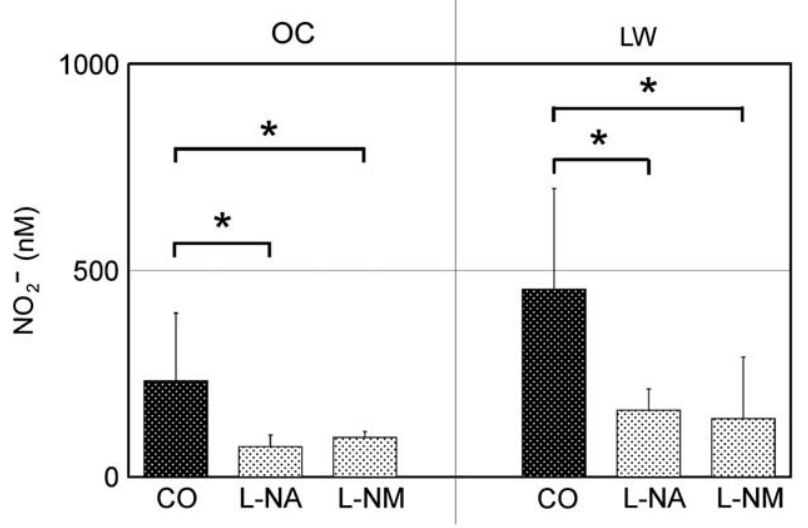

Fig. 3 Up- and downregulation of NO production in cochlear organ cultures. a The NO production was clearly upregulated in the organ of Corti after DETA application using a concentrations of $50 \mu \mathrm{M}$ and $500 \mu \mathrm{M}$ and in the lateral wall using a concentration of $500 \mu \mathrm{M}$. b The application of L-NAME (L-NA) or L-NMMA (L-NM) reduced the NO production in the organ of Corti and in the lateral wall.

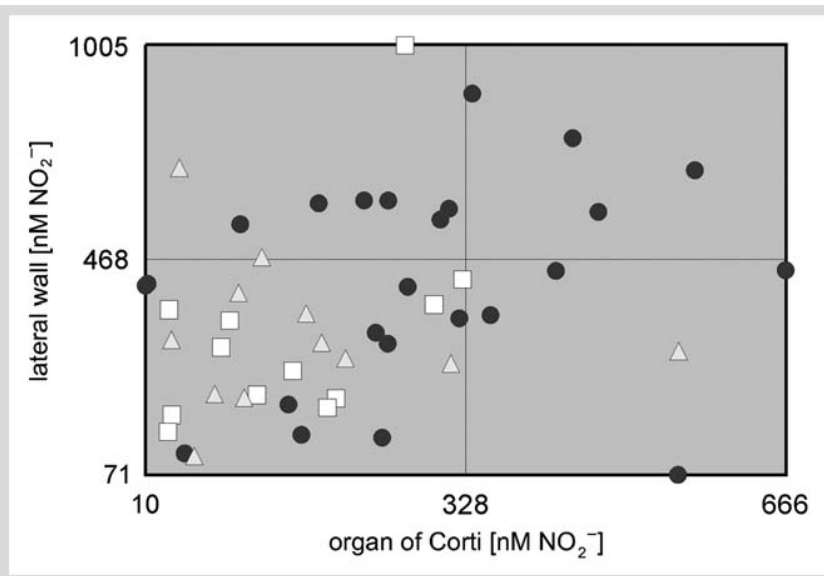

Fig. 4 Scatter plot of the values of $\mathrm{NO}_{2}{ }^{-}$production in the organ of Corti and the lateral wall for each control ear (solid circle) and $17 \beta$-estradiol-treated ears of group B (open squares) and group C (triangles). The values were separated by lines into a "low" group if their values were closer to the minimum than to the maximum and otherwise to a "high" group. Nearly all $17 \beta$-estradiol-treated ears exhibited lower values, with $\mathrm{NO}_{2}{ }^{-}$production below $468 \mathrm{nM}$ in the lateral wall and below $328 \mathrm{nM}$ in the organ of Corti.

\section{Discussion and Conclusion}

$\nabla$

In this experimental animal study, the influence of $17 \beta$-estradiol on NO production was tested in organ cultures of the guinea pig cochlea. In addition, the system was evaluated by the NO donor DETA and the NOS inhibitors L-NAME and L-NMMA. The basal mean NO production in cultures of the organ of Corti was clearly reduced by $17 \beta$-estradiol, whereas the average NO production in the lateral wall was only marginally lowered. When comparing the values of NO production in both regions within the same cochlea, a clear association becomes evident. Low values of NO production were predominately measured in the groups, which were treated by $17 \beta$-estradiol compared with controls. Thus, an NO-reducing effect of $17 \beta$-estradiol was clearly demonstrated for the organ of Corti. In both cochlear regions, an increase in NO production was achieved by DETA and a decrease by L-NAME and L-NMMA. Furthermore, it was also shown that no alterations in cellular morphology were seen in the electron microscope after a $6 \mathrm{~h}$ incubation period.

Analyzing the average values of the NO production in both the organ of Corti and the lateral wall, it became evident that there was a great variability in each group. Comparable variations in NO production were found in other experiments [25-27], which are based on the natural inter-animal differences in inner ear physiology, especially of guinea pigs [28]. Organ cultures are widely used in inner ear research to identify cellular physiological pathways $[23,24]$. Earlier studies already demonstrated that explants of the stria vascularis and spiral ligament dissected from the guinea pig cochlea could be successfully cultivated for several weeks [29]. Unfortunately, the culture technique through extraction of tissues from the bony capsule leads to the loss of structural preservation thus hampering further morphological analyses or histological examinations. Despite this disadvantage, the present study confirmed the integrity of cellular morphology at the electron microscopic level.

$17 \beta$-Estradiol is a naturally produced hormone and was shown to reduce the inner ear NO production predominately in the organ of Corti. Comparable effects were demonstrated for other pharmacological substances. A noise-induced increase in NO production could be prevented in both the organ of Corti and the lateral wall when ascorbic acid was supplemented over a period of 7 days prior to noise exposure [25]. In addition, a reduction in tissue-related NO production within organ cultures of both the organ of Corti and the lateral wall was demonstrated by the application of doxycycline [30]. Doxycycline and its related minocycline both belong to the semi-synthetic second-generation tetracyclines, which possess anti-inflammatory effects that are completely distinct from their antimicrobial actions [31]. The comprehensive NO reduction was comparable to the effect of the nitric oxide synthase (NOS) inhibitors $\mathrm{N}^{\mathrm{G}}$-Nitro-L-arginine methyl ester (L-NAME) and NG-monomethyl-L-arginine monoacetate (L-NMMA) [30]. An increase in NO production was measured in the lateral wall after intratympanic application of gentamicin, an aminoglycoside, which is commonly used in the treatment of Ménière's disease [32]. This alteration was exclusively restricted to the lateral wall, whereas the NO production in the organ of Corti remained nearly unaltered [26]. Thus, the various findings demonstrate that NO production in the organ of Corti and the lateral wall can be differently influenced by various stimuli resulting in an increase or decrease respectively. The comparable analysis by the NO donor DETA or the NOS inhibitors L-NAME and L-NMMA, respectively, confirmed the ability to 
selectively influence the NO production in cochlear tissues, the organ of Corti and the lateral wall.

The adequate regulation of $\mathrm{NO}$ amount is important for the precise controlling of physiological pathways [33]. Generally, the reduction of NO production by $17 \beta$-estradiol in the organ of Corti could be evoked by 2 mechanisms - a spatial-specific downregulation of the constitutively expressed NOS isoforms and/or a reduction of its activity via blockage of $\mathrm{Ca}^{2+}$ transporters. To the best of our knowledge there is currently no report about the direct downregulation in the expression of eNOS or the neuronal nitric oxide synthase (nNOS) by $17 \beta$-estradiol. It is commonly known that the activity of the constitutive NOS isoforms, the eNOS and the nNOS, are dependent on intracellular $\mathrm{Ca}^{2+}$ concentration. Therefore, a $17 \beta$-estradiol-mediated downregulation of NOS activity might exist in the organ of Corti as identified so far in the uterus and vagina in rabbits [13].

Two different $\mathrm{Ca}^{2+}$ transporters are generally involved in the blockage process - the $\mathrm{Ca}^{2+}$ channels and the $\mathrm{Na}^{+}-\mathrm{Ca}^{2+}$ exchanger. A $17 \beta$-estradiol-dependent blockage of L-type $\mathrm{Ca}^{2+}$ channels was reported for murine cardiomyoctes [14] and VSC 4.1 cell line [15]. It is assumed that $17 \beta$-estradiol leads to a direct change of the pore conformation close to the external mouth of the L-type channels, a closure of the channels in their open state and/or modulation of channel function by estrogen-induced phosphorylation [34]. In the organ of Corti, $\mathrm{Ca}^{2+}$ channels were identified in sensory and supporting cells with variable expression patterns [35-38]. In the lateral wall, $\mathrm{Ca}^{2+}$ channels were detected so far in the marginal cells $[37,39]$.

A $17 \beta$-estradiol-dependent influence on the $\mathrm{Na}^{+}-\mathrm{Ca}^{2+}$ exchanger was reported for mouse myocytes [40]. Furthermore, 17ß-estradiol produced an increase in the $\mathrm{Na}^{+}-\mathrm{Ca}^{2+}$ exchanger-mediated inward current and a decrease in the outward current in rat cultured primary cortical neurons. The blockade resulted in a decreased $\mathrm{Ca}^{2+}$ influx and increased $\mathrm{Ca}^{2+}$ efflux [41]. In the vertebrate cochlea, the $\mathrm{Na}^{+}-\mathrm{Ca}^{2+}$ exchanger was identified in the outer hair cells [42].

From the present state of knowledge, it is evident that there is a quantitative divergence in the amount of calcium transport systems between the organ of Corti and the lateral wall. Because of that, it is hypothesized that the significant NO downregulation in the organ of Corti is primarily based on the higher number of $\mathrm{Ca}^{2+}$ transporters, which became blocked by $17 \beta$-estradiol.

\section{Acknowledgements}

The authors thank Mrs. K. Benz for her invaluable technical assistance.

\section{Conflict of Interest}

The authors declare that they have no conflicts of interest in the authorship or publication of this contribution.

\section{References}

1 Takumida M, Anniko M. Nitric oxide in the inner ear. Curr Opin Neurol 2002; 15: 11-15

2 Pacher P, Beckman JS, Liaudet L. Nitric oxide and peroxynitrite in health and disease. Physiol Rev 2007; 87: 315-424

3 Heinrich UR, Helling $K$. Nitric Oxide - A versatile key player in cochlear function and hearing disorders. Nitric Oxide 2012; 27: 106-116
4 Watanabe K, Hess A, Zumeger C, Stennert E, Bloch W, Addicks K, Michel $O$. Changes of the compound action potential (CAP) and the expression of inducible nitric oxide synthase (iNOS/NOS II) in the cochlea under the inflammatory condition. Hear Res 2000; 145: 149-155

5 Shi X, Ren T, Nuttall AL. The electrochemical and fluorescence detection of nitric oxide in the cochlea and its increase following loud sound. Hear Res 2002; 164: 49-58

6 Shi X, Nuttall AL. Upregulated iNOS and oxidative damage to the cochlear stria vascularis due to noise stress. Hear Res 2003; 967: 1-10

7 Pall ML, Bedient SA. The NO/ONOO- cycle as the etiological mechanism of tinnitus. Int Tinnitus J 2007; 13: 99-104

8 Chen YS, Tseng FY, Lin KN, Yang TH, Lin-Shiau SY, Hsu CJ. Chronologic changes of nitric oxide concentration in the cochlear lateral wall and its role in noise-induced permanent threshold shift. Laryngoscope 2008; 118: 832-836

9 Nagura M, Iwasaki S, Wu R, Mizuta K, Umemura K, Hoshino T. Effects of corticosteroid, contrast medium and ATP on focal microcirculatory disorders of the cochlea. Eur J Pharmacol 1999; 366: 47-53

10 Chandrasekhar SS. Intratympanic dexamethasone for sudden sensorineural hearing loss: clinical and laboratory evaluation. Otol Neurotol 2001; 22: 18-23

11 Gouveris $H$, Selivanova O, Mann W. Intratympanic dexamethasone with hyaluronic acid in the treatment of idiopathic sudden sensorineural hearing loss after failure of intravenous steroid and vasoactive therapy. Eur Arch Otorhinolaryngol 2005; 262: 131-134

$12 \mathrm{Kim} \mathrm{KH}$, Bender JR. Membrane-initiated actions of estrogen on the endothelium. Mol Cell Endocrinol 2009; 308: 3-8

13 Batra S, Al-Hijji J. Characterization of nitric oxide synthase activity in rabbit uterus and vagina: downregulation by estrogen. Life Sci 1998; 62: 2093-2100

14 Ullrich ND, Krust A, Collins P, MacLeod KT. Genomic deletion of estrogen receptors $E R \alpha$ and ER $\beta$ does not alter estrogen-mediated inhibition of $\mathrm{Ca}^{2+}$ influx and contraction in murine cardiomyocytes. Am J Physiol Heart Circ Physiol 2008; 294: H2421-H2427

15 Sribnick EA, Del Re AM, Ray SK, Woodward JJ, Banik NL. Estrogen attenuates glutamate-induced cell death by inhibiting $\mathrm{Ca}^{2+}$ influx through L-type voltage-gated $\mathrm{Ca}^{2+}$ channels. Brain Res 2009; 1276: 159-170

16 Manthey D, Behl C. From structural biochemistry to expression profiling: neuroprotective activities of estrogen. Neurosci 2006; 138: 845-850

17 Singh $M$, Dykens JA, Simpkins JW. Novel mechanisms for estrogeninduced neuroprotection. Exp Biol Med 2006; 231: 514-521

18 Brann DW, Dhandapani K, Wakade C, Mahesh VB, Khan MM. Neurotrophic and neuroprotective actions of estrogen: basic mechanisms and clinical implications. Steroids 2007; 72: 381-405

19 Suzuki S, Brown CM, Wise PM. Neuroprotective effects of estrogens following ischemic stroke. Front Neuroendocrinol 2009; 30: 201-211

20 Hultcrantz M, Simonoska R, Stenberg AE. Estrogen and hearing: a summary of recent investigations. Acta Otolaryngol 2006; 126: 10-14

21 Motohashi R, Takumida M, Shimizu A, Konomi U, Fujita K, Hirakawa $K$, Suzuki M, Anniko M. Effects of age and sex on the expression of estrogen receptor $\alpha$ und $\beta$ in the mouse inner ear. Acta Otolaryngol 2010; 130: 204-214

22 Chow RW, Handelsman DJ, $\mathrm{Ng}$ MK. Minireview: Rapid actions of sec steroids in the epithelium. Endocrinol 2010; 151: 2411-2422

23 Malgrange B, Rigo JM, Coucke P, Thiry M, Hans G, Nguyen L, van de Water TR, Moonen G, Lefebvre PP. Identification of factors that maintain mammalian outer hair cells in adult organ of Corti explants. Hear Res 2002; 170: 48-58

24 Moriyama M, Yoshida K, Ichimiya I, Suzuki M. Nitric oxide production from cultured spiral ligament fibrocytes: effects of corticosteroids. Acta Otolaryngol 2007; 127: 676-681

25 Heinrich UR, Fischer I, Brieger J, Rümelin A, Schmidtmann I, Li H, Mann $W J$, Helling K. Ascorbic acid reduces noise-induced nitric oxide production in the guinea pig ear. Laryngoscope 2008; 118: 837-842

26 Heinrich UR, Helling K, Sifferath M, Brieger J, Li H, Schmidtmann I, Mann WJ. Gentamicin increases NO production and induces hearing loss in guinea pigs. Laryngoscope 2008; 118: 1438-1442

27 Helling K, Heinrich UR, Heusgen L, Brieger J, Schmidtmann I, Li H, Mann WJ. Gentamicin alters nitric oxide production in semicircular canals and otolith organs. Laryngoscope 2010; 120: 2125-2128

28 Wang Y, Hirose K, Liberman MC. Dynamics of noise-induced cellular injury and repair in the mouse cochlea. JARO 2002; 3: 248-268

29 Melichar I, Gitter AH. Primary culture of vital marginal cells from cochlear explants of the stria vascularis. Eur Arch Otorhinolaryngol 1991; 248: 358-365 
30 Helling K, Wodarzcyk K, Brieger J, Schmidtmann I, Li H, Mann WJ, Heinrich UR. Doxycycline reduces nitric oxide production in guinea pig inner ears. Auris Nasus Larynx 2011; 38: 671-677

31 Ryan ME, Ashley RA. How do tetracyclines work? Adv Dent Res 1998; 12: 149-151

32 Helling K, Schönfeld U, Clarke AH. Treatment of Ménière's disease by low-dosage intratympanic gentamicin application: effect on otolith function. Laryngoscope 2007; 117: 2244-2250

33 Thomas DD, Ridnour LA, Isenberg JS, Flores-Santana W, Switzer CH, Donzelli S, Hussain P, Vecoli C, Paolocci N, Ambs S, Colton CA, Harris CC, Roberts DD, Wink DA. The chemical biology of nitric oxide: implications in cellular signalling. Free Rad Biol Med 2008; 45: 18-31

34 Fatehi M, Kombian SB, Saleh TM. 17ß-estradiol inhibits outward potassium currents recorded in rat parabrachial nucleus cells in vitro. Neuroscience 2006; 135: 1075-1086

35 Raybould NP, Jagger DJ, Kanjhan R, Greenwood D, Laslo P, Hoya N, Soeller C, Cannell MB, Housley GD. TRPC-like conductance mediates restoration of intracellular $\mathrm{Ca} 2+$ in cochlear outer hair cells in the guinea pig and rat. J Physiol 2007; 579 (Pt 1): 101-113

36 Mammano F, Bortolozzi M, Ortolano S, Anselmi F. $\mathrm{Ca}^{2+}$ signalling in the inner ear. Physiol 2007; 22: 131-144
37 Takumida M, Ishibashi T, Hamamoto T, Hirakawa K, Anniko M. Agedependent changes in the expression of klotho protein, TRPV5 and TRPV6 in mouse inner ear. Acta Otolaryngol 2009; 129: 1340-1350

38 Yamauchi $D$, Nakaya K, Raveendran NN, Singh $R$, Wangemann P, Marcus $D C$. Expression of epithelial calcium transport system in rat cochlea and vestibular labyrinth. BMC Physiol 2010; 10: 1

39 Inui T, Mori Y, Watanabe M, Takamaki A, Yamaji J, Sohma Y, Yoshida $R$, Takenaka H, Kubota T. Physiological role of L-type $\mathrm{Ca}^{2+}$ channels in marginal cells in the stria vascularis of guinea pigs. J Physiol 2007; 57: $287-298$

40 Sugishita K, Su Z, Li F, Philipson KD, Barry WH. Gender influences $[\mathrm{Ca}(2+)](\mathrm{i})$ during metabolic inhibition in myocytes overexpressing the $\mathrm{Na}(+)-\mathrm{Ca}(2+)$ exchanger. Circulation 2001; 104: 2101-2106

41 Sánchez JC, López-Zapata DF, Francis L, Los Reyes LD. Effects of estradiol and IGF-1 on the sodium calcium exchanger in rat cultured cortical neurons. Cell Mol Neurobiol 2011; 31: 619-627

42 Surin AM, Reimann-Philipp U, Fechter LD. Simultaneous monitoring of slow cell motility and calcium signals of the guinea pig outer hair cells. Hear Res 2000; 146: 121-133 